\title{
Tuning the viscoelastic features required for 3D printing of PVC-acrylate copolymers obtained by single electron transfer-degenerative chain transfer living radical polymerization (SET-DTLRP)
}

\author{
M. I. Calafel ${ }^{1}$, R. H. Aguirresarobe ${ }^{1}$, N. Sadaba ${ }^{2}$, M. Boix ${ }^{3}$, J. I. Conde C B. Pascual $^{3}$, \\ A. Santamaria ${ }^{*}$ \\ ${ }^{1}$ POLYMAT and Polymer Science and Technology Department, Faculty of Chemistry, UPV/EHU, Avda. Tolosa 72, \\ 20018 San Sebastian, Spain \\ ${ }^{2}$ POLYMAT and Mining-Metallurgy Engineering and Material Science Department, Faculty of Engineering, UPV/EHU, \\ Alameda de Urquijo s/n, 4013 Bilbao, Spain \\ ${ }^{3}$ ERCROS S.A., Innovation and Technology Department, Chlorine Derivatives Division, Diagonal 595, 08014 Barcelona, \\ Spain
}

Received 1 March 2018; accepted in revised form 2 May 2018

\begin{abstract}
Random poly (vinyl chloride-co-butyl acrylate) and poly (vinyl chloride-co-2-ethylhexyl acrylate copolymers obtained by single electron transfer-degenerative chain transfer living radical polymerization (SET- DTLRP) are investigated as potential candidates for 3D Printing. The analysis of the rheological implications of 3D Printing process allows establishing the basic viscoelastic conditions that the samples should fulfil to be printable, avoiding the 'trial and error' procedure. The effect of temperature and acrylates concentration on the rheological properties and 3D printing feasibility is contemplated. Eventually, thermal degradation is also considered. It is demonstrated that the copolymers which contain butyl acrylate comonomer, instead of 2-ethylhexyl acrylate, give raise to the best results. Although the study is carried out with PVCacrylate copolymers and a 3D printing device that possesses set up characteristics, it shows a way to work out sound strategies with other polymers and 3D printing machines.
\end{abstract}

Keywords: rheology, processing technologies, 3d-printing, dynamic viscoelasticity, pvc-acrylate copolymers

\section{Introduction}

Additive manufacturing techniques have attracted attention since they exhibit versatility in the rapid fabrication of prototypes and complex shapes $[1,2]$. In addition, it follows the 'Do it yourself' (DIY) trend, which aims to reduce costs in manufacturing processes [3]. Among different additive techniques, melt-extrusion additive manufacturing techniques have been proposed as one of the most common devices due to the easy maintenance and reduce cost [1]. Melt-extrusion additive manufacturing techniques includes fused filament fabrication (FFF) as well as extrusion based printing techniques $[4,5]$. Although the first alternatives are the most employed ones, extrusion based techniques present several advantages as the higher dimensional accuracy and the ability to work at lower pressures. In addition, from an operational point of view, this technique resembles conventional extrusion, as printer is fed with polymer pellets, which avoid the pre-extrusion of the material required in filament based devices. Such characteristic potentially permits to expand the number of materials 
suitable for 3D printing, which are, at the moment, rather limited.

However, the implementation of extrusion based 3D printing techniques has not grown as desired, mainly by the difficulties of incorporating polymer commodities to such techniques. This is the case of PVC derivatives, which are one of the most used materials nowadays, in common applications ranging from building industry to packaging [6]. However, PVC has not been implemented for 3D printing techniques and very few examples of printing of PVC can be found in literature at the moment, all of them regarding fused filament fabrication (FFF) techniques. To the best of our knowledge, Aoniq (Mitchell, Australia) has developed a specific FFF device for PVC and Chemson (Arnoldstein, Austria) has produced PVC filaments although the approach focuses on the industrial point of view. The main reason for this scenario could be related to the difficulty of PVC and their derivatives to fulfil the requirements established in melt-extrusion 3D printing processes. Thus, the understanding of the underlying rheological parameters of materials required for a successful printing, which are unclear at the moment and forces manufacturers to a trial-and-error process, can help to expand the number of materials suitable for these manufacturing techniques.

Several studies focus on the modelling of melt-extrusion additive manufacturing, both fused filament fabrication $[4,7]$, and extrusion-based printing techniques $[8,9]$. In essence, $3 \mathrm{D}$ printing using polymer melts implies at least three essential features:

a) Adequate viscosity to ensure the envisaged printing velocity

b) Immediate adhesion of deposed polymer layers and

c) Adequate cooling process to guarantee final mechanical properties [10-12].

In general terms, increasing temperature allows getting suitable viscosities and layer adhesion. However, besides too low viscosity, which can lead to a mismatch of the exit velocity at the needle with the printing velocity, high temperatures can also give rise to polymer degradation. This is a general issue, but it is particularly relevant for PVC samples, which at temperatures above $150^{\circ} \mathrm{C}$ are only thermally stable for relatively short times [13]. Therefore, the thermal behaviour of PVC should be contemplated when dealing with $3 \mathrm{D}$ printing of this material.
This work aims to establish some rheological criteria that PVC derivatives should accomplish to be good candidates for extrusion-based 3D printing. In order to do so, we study the rheological features of different conventional and new PVC formulations, from rigid to flexible materials. Regarding the flexible materials, this study takes advantage of recently developed selfplasticised PVCs on the basis of random poly (vinyl chloride-co-butyl acrylate) and poly (vinyl chlorideco-2-ethylhexyl acrylate) copolymers obtained by single electron transfer-degenerative chain transfer living radical polymerization (SET-DTLRP) [14, 15]. SET-DTLRTP technique is a valuable synthetic alternative for PVC derivatives as it permits to face problems derived from the synthesis of this polymer [1619]. Additionally, the controlled character of this technology permits the synthesis of well-defined copolymers, from block to random structures, using diverse comonomers $[14,15,20,21]$. The selected materials offer the advantage of having low glass transition temperatures, as compared to PVC, which facilitates extrusion and adhesion between layers during additive manufacturing.

\section{Experimental part}

\subsection{Materials}

Poly(vinyl chloride) (PVC) and poly(vinyl chlorideco-butyl acrylate) (PVC-BA) and poly(vinyl chloride-co-2-ethylhexyl acrylate) (PVC-EHA) copolymers were obtained by single electron transfer-degenerative chain transfer living radical polymerization (SET-DCTLRP) as described elsewhere [14, 15]. Additionally, reference plasticized PVC-DOP samples were obtained by melt mixing of the previously used PVC with the appropriate amounts of bis(2-ethylhexyl)phthalate (DOP).

The composition of the copolymers was evaluated by proton nuclear magnetic resonance ( $\left.{ }^{1} \mathrm{H}-\mathrm{NMR}\right)$ spectra recorded in a Bruker Analytische Messetechnik 500, at $500.13 \mathrm{MHz}$ of resonance frequency. The band assignment was conducted following the criteria of our previous works $[14,15]$, and the lines between 4 and $5 \mathrm{ppm}$ (corresponding to $-\mathrm{CO}-\mathrm{O}-\mathrm{CH}_{2}-$ ) were used to calculate the composition of copolymers. Additionally, the microstructures of different copolymers were confirmed by ${ }^{13} \mathrm{C}-\mathrm{NMR}$, as it is explained in reference [15]. The materials used, as well as the PVC, DOP and PBA amounts of each material, calculated by ${ }^{1} \mathrm{H}-\mathrm{NMR}$, are summarized in Table 1 . 
Table 1. Materials used in the study and the relative amounts of PVC, DOP and acrylate in each sample.

\begin{tabular}{|l|c|c|c|}
\hline \multicolumn{1}{|c|}{ Sample } & $\begin{array}{c}\text { PVC } \\
{[\mathbf{w t} \%]}\end{array}$ & $\begin{array}{c}\text { DOP } \\
{[\mathbf{w t} \% \mathbf{~}]}\end{array}$ & $\begin{array}{c}\text { Acrylate } \\
{[\mathbf{w t} \% \mathbf{~}]}\end{array}$ \\
\hline PVC & 100 & - & - \\
\hline PVC-65DOP & 61 & 39 & - \\
\hline PVC-BA Block & 50 & - & 50 \\
\hline \multicolumn{4}{|c|}{ PVC-BA Random } \\
\hline PVC-20\% BA & 80 & - & 20 \\
\hline PVC-30\% BA & 70 & - & 30 \\
\hline PVC-40\% BA & 62 & - & 38 \\
\hline PVC-50\% BA & 53 & - & 47 \\
\hline \multicolumn{4}{|c|}{ PVC-EHA Random } \\
\hline PVC-20\% EHA & 77 & - & 23 \\
\hline PVC-30\% EHA & 70 & - & 30 \\
\hline PVC-40\% EHA & 62 & - & 38 \\
\hline PVC-50\% EHA & 52 & - & 48 \\
\hline
\end{tabular}

\subsection{Thermal analysis}

Dynamic mechanical thermal analysis (DMTA) measurements were carried out in a Dynamic Mechanical Analyzer, Triton 2000 DMA from Triton Technology (Mansfield, UK), in bending mode. The samples were heated from 10 to $120^{\circ} \mathrm{C}$ at a constant heating rate of $4^{\circ} \mathrm{C} / \mathrm{min}$ and a frequency of $1.0 \mathrm{~Hz}$; this allowed detecting the glass transition temperature $\left(T_{\mathrm{g}}\right)$, given by a maximum peak in loss tangent, $\tan \delta=G^{\prime \prime} / G^{\prime}$.

Since crystallinity is a key point for eventual shrinkage or warpage effects during cooling, the degree of crystallinity of all the samples was measured by differential scanning calorimetry (DSC) in a Q-2000 (TA instruments, New Castle, DE, USA) under nitrogen, using a scan rate of $10^{\circ} \mathrm{C} / \mathrm{min}$ in the temperature range 50 to $200^{\circ} \mathrm{C}$. Neat PVC, as well as PVC 65DOP and PVC-50\% BA block copolymer showed crystallinities between 7 and 9\% (referred to vinyl chloride content in the sample). However, the crystallinity of random copolymers was almost negligible. These results are in good agreement with the different microstructure of the synthesized copolymers. Samples containing PVC domains (neat PVC, PVC 65DOP and PVC-BA block copolymer) present a crystalline phase. In contrast, the microstructure obtained for random copolymers, where no PVC phase was observed, impedes the formation of PVC crystals.

The thermogravimetric analysis of the samples was conducted in a TA Q500 device (TA instruments, New Castle, DE, USA) in isothermal mode.

\subsection{Rheological measurements}

Dynamic viscoelastic measurements were carried out using an AR-G2 rheometer (TA instruments, New Castle, DE, USA) with a parallel-plate fixture (25 mm diameter), conducting oscillatory frequency sweep experiments in the linear regime at the temperatures indicated in the Results and Discussion section. The shear elastic modulus, $G^{\prime}$, shear loss modulus, $G^{\prime \prime}$, and the complex viscosity, $\left|\eta^{*}(\omega)\right|=\sqrt{\left(G^{\prime} / \omega\right)^{2}+\left(G^{\prime \prime} / \omega\right)^{2}}$, were evaluated as a function of frequency. The absolute value of the complex viscosity was used as representative of the viscosity obtained in oscillatory flow.

Extrusion flow experiments were performed in a Göttfert 2002 rheometer (Buchen, Germany) using a capillary die with $L / D=30 / 1$, at the temperatures and shear rates indicated in Results and Discussion section. The viscosity curves, i.e. viscosity as a function of shear rate, $\eta(\dot{\gamma})$, were obtained.

\subsection{D Printer and optical analysis of the samples}

The printing process was carried out in a 3D-Bioplotter (Developer Series, EnvisionTEC, Gladbeck, Germany) and the Printing geometries were originally designed in Solidworks 2016 x64 Editor. Typically the printing of the samples was performed at a temperature of $160^{\circ} \mathrm{C}$, with $0.001 \mathrm{~mm}$ of resolution, and a maximum pressure of $8.8 \cdot 10^{5}$ Pascals. In this study, needles with an inner diameter of $0.4 \mathrm{~mm}$ were used.

Leitz Aristomet polarized light optical microscope (Wetzlar, Germany) with a Leitz Wetzlar L20/0.32 objective was used to have an inside of the welding of printed materials.

\section{Results and discussion \\ 3.1. Rheological characterization of PVC-acrylate copolymers}

In order to study the potential 3D printing characteristics of our materials, we first analysed their rheological behaviour. Thus, continuous extrusion flow (for the high shear rates region) and oscillatory measurements under linear viscoelastic conditions were used to characterize the rheological behaviour of the samples. Figures $1 \mathrm{a}$ and $1 \mathrm{~b}$ show, respectively, the absolute value of the complex viscosity $\eta^{*}(\omega)$ obtained by oscillatory flow and the viscosity function $\eta(\dot{\gamma})$ 

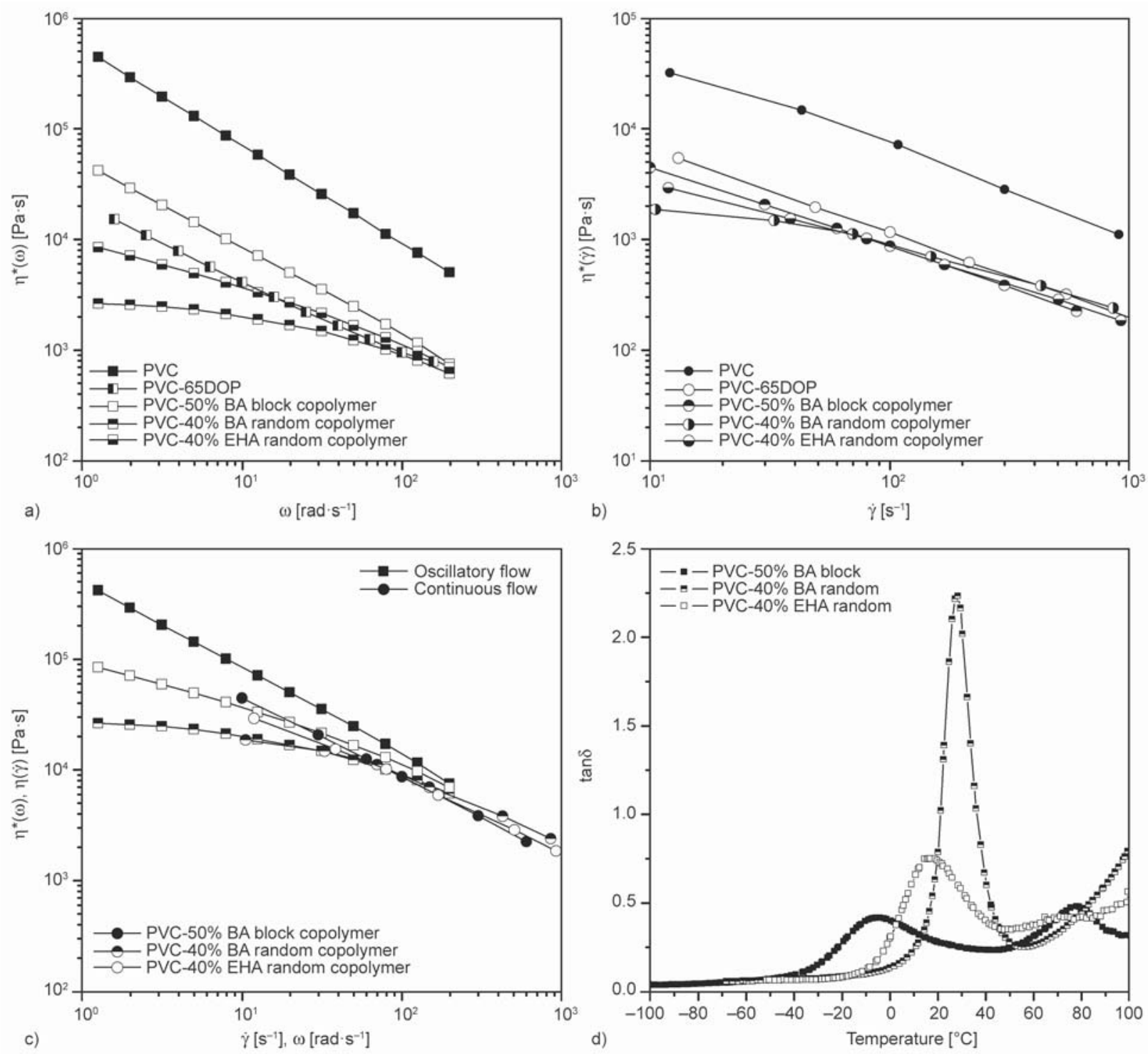

Figure 1. Viscosity and DMTA results for the analysed PVC derivatives at $160^{\circ} \mathrm{C}$. a) Complex viscosity vs. frequency, b) continuous flow viscosity versus shear rate, c) overlapped plots of block and random copolymers, as proposed in Equation (1), d) DMTA results for the analysed copolymers.

obtained by extrusion flow, for the analysed samples. As expected, all samples showed shear thinning behaviour with a clear plasticizing effect for the proposed copolymers with respect to the neat PVC. In order to compare both, oscillatory and continuous extrusion flow viscosities, and to refer to the 3D printing parameters that will be discussed later, the results were analysed considering the empiric CoxMerz rule [22, 23], described in Equation (1):

$$
\left|\eta^{*}(\omega)\right|=\sqrt{\eta^{\prime}(\omega)^{2}+\eta^{\prime \prime}(\omega)^{2}}=\left.\eta(\dot{\gamma})\right|_{\dot{\gamma}=\omega}
$$

where the frequency is expressed in $\mathrm{rad} / \mathrm{s}$ and the shear rate in $1 / \mathrm{s}$.

As can be seen in 1c, PVC-40\% BA random copolymer obeys the Cox-Merz rule resulting in the overlap of the continuous flow viscosity, $\eta(\dot{\gamma})$ and the absolute value of the oscillatory flow complex viscosity $\eta^{*}(\omega)$. This is not the case of PVC-50\% BA block copolymer which shows very different values of $\eta^{*}(\omega)$ and $\eta(\dot{\gamma})$ in the plots of Figure 1c. For PVC$40 \%$ EHA random copolymer both viscosities lie close, but the Cox-Merx rule is not strictly satisfied, as compared to PVC- $40 \%$ BA.

Actually, it has been observed that Cox-Merz rule is only fulfilled for homogeneous polymer samples [22]. Thus, the results of Figure 1c confirm the homogeneous nature of our PVC-BA random copolymers, which is compatible with an equal concentration of monomers in all the chains, as was remarked in a previous paper [15]. The analysis of the eventual matching of $\eta^{*}(\omega)$ and $\eta(\dot{\gamma})$ data in PVC-50\% BA 
block copolymer, PVC-40\% BA random copolymer and PVC- $40 \%$ EHA random copolymer, is completed with DMTA results depicted in Figure 1d. As it could be expected, PVC-50\% BA block copolymer shows two glass transition temperatures, indicating two separated phases, whereas PVC- $40 \%$ BA random copolymer presents only one glass transition temperature, denoting a single phase. These results are compatible with those observed in Figure 1c: Only the single phase homogeneous copolymer fulfils the Cox-Merx rule. The case of PVC- $40 \%$ EHA random copolymer is in between these two contrasting behaviours. It can be stated that this is not actually a neat random copolymer, since it shows a main $T_{\mathrm{g}}$ close to $20^{\circ} \mathrm{C}$ and a smaller $T_{\mathrm{g}}$ peak around $70^{\circ} \mathrm{C}$. This second glass transition temperature can be attributed to small PVC domains, which, in turn, results in a not straight accomplishment of Cox-Merz equation for this sample.

\subsection{Rheological conditions for printing}

To contextualize our viscosity results to the framework of the operation conditions of the 3D Printer, we first analyse the rheological parameters of the flow in the needle. The approach is made on the assumption of a polymer melt which satisfies the power law equation $\eta=k \dot{\gamma}^{\mathrm{n}-1}$, where $n<1$ is the shear thinning index [22]. In this case, the shear rate in the wall of the needle, $\dot{\gamma}$, is directly proportional to the exit velocity, $v$, of the polymer, as represented in Equation (2):

$\dot{\gamma}=\frac{3 n+1}{4 n} \cdot \frac{4 v}{R}$

where $R$ is the radius of the needle.

Provided that to accomplish a layer of the diameter of the needle the exit velocity should coincide with the printing velocity, Equation (1) provides us the value of the shear rate for each printing velocity. Considering and average value of $n=0.3$ and attending to the characteristics of our 3D Printing machine (see Experimental Part) for a needle of diameter $0.4 \mathrm{~mm}$, the minimum shear rate is $\dot{\gamma}=3.2 \mathrm{~s}^{-1}$ for the minimum printing velocity, $0.1 \mathrm{~mm} / \mathrm{s}$, whereas the maximum printing velocity $150 \mathrm{~mm} / \mathrm{s}$ gives a maximum shear rate of $4750 \mathrm{~s}^{-1}$.

On the other hand, the pressure, $P$, applied to make the melt flow through the needle is directly related to the shear stress at the wall of the needle by equation (3) [22]:
$\sigma=P \cdot \frac{D}{4 L}$

where $D$ and $L$ are, respectively, the diameter and length of the needle.

The extreme conditions correspond to the maximum allowable pressure which is $P=8.8 \cdot 10^{5} \mathrm{~Pa}$. Since our experiments are performed with a needle of $D=$ $0.4 \mathrm{~mm}$ and $L=8.9 \mathrm{~mm}$, according to Equation (3), the estimated maximum shear stress is $9.9 \cdot 10^{3} \mathrm{~Pa}$. Then the viscosities, $\eta=\sigma / \dot{\gamma}$ which correspond to printing velocities of 0.1 (shear rate of $\dot{\gamma}=3.2 \mathrm{~s}^{-1}$ ) and $150 \mathrm{~mm} / \mathrm{s}$ (shear rate of $\dot{\gamma}=4750 \mathrm{~s}^{-1}$ ) are, respectively, 3100 and $2 \mathrm{~Pa} \cdot \mathrm{s}$, for an applied pressure of $8.8 \cdot 10^{5} \mathrm{~Pa}$. Combining Equations (2) and (3) it can be seen that for a constant pressure the viscosity is inversely proportional to the shear rate and, consequently, to the exit velocity. This result allows drawing a line in a viscosity-shear rate plot, above which the viscosity is excessively high to grant $3 \mathrm{D}$ printing, even at the highest applied pressure $P=8.8 \cdot 10^{6} \mathrm{~Pa}$, as it is shown in Figure 2.

In this framework, the viscosity of the materials was studied to establish the potential 3D printability of different PVC derivatives. In Figure 2, the complex viscosity $\eta^{*}(\omega)$ is used as representative to cover viscosity values below shear rates $10 \mathrm{~s}^{-1}$, at which $\eta(\dot{\gamma})$ was not available by continuous extrusion flow. This is a valid approach for both random copolymers,

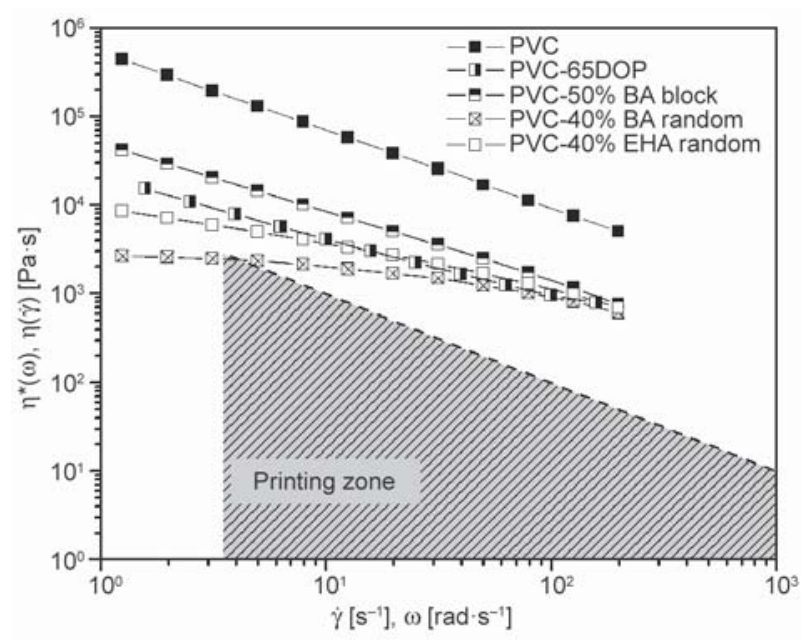

Figure 2. Complex viscosity, $\eta^{*}(\omega)$ as a function of frequency at $T=160^{\circ} \mathrm{C}$ for the investigated samples. The line marks the postulated continuous flow viscosity $\eta(\dot{\gamma})$ as a function of the shear rate for the maximum pressure of $8.8 \cdot 10^{5} \mathrm{~Pa}$ in the $3 \mathrm{D}$ printing machine (see text). For viscosities above the line which marks the 'printing zone', 3D printing is not possible. 
according to the results of Figure 1c. In the case of the other samples, PVC, PVC-65DOP and PVC$50 \%$ BA block copolymer, Cox-Merz rule is not fulfilled, but the complex viscosity $\eta^{*}(\omega)$ can be used as a reference, considering that the values of $\eta(\dot{\gamma})$ are given in Figure $1 \mathrm{~b}$ for comparison purposes.

Taking into account the required conditions for 3D printing, stated above and marked by the line and the 'printing zone' in Figure 2, it can be predicted that neat PVC, PVC-DOP as well as PVC-50\% BA block copolymer should be excluded for 3D printing in our equipment, as they present viscosity values higher than those required for the extrusion through the needle. This exclusion is also deduced considering the $\eta(\dot{\gamma})$ viscosity values shown for these samples in Figure $1 \mathrm{~b}$, which are clearly above the marked 'printing zone'. On the contrary, the viscosity of PVC- $40 \%$ BA random copolymer enters in this zone, while the values for PVC-40\% EHA random copolymer are out of the limits of polymer extrusion. Experimental 3D printing tests, which are discussed more extensively below, show that neat PVC, PVC-DOP, PVC$50 \%$ BA block copolymer and PVC-40\% EHA random copolymer are not printable, whereas PVC$40 \%$ BA random copolymer is adequate for printing. This demonstrates that viscosity measurements can constitute a valuable tool to avoid trial and error processes in additive manufacturing.

\subsection{Effect of temperature}

The effect of temperature on the 3D printing performance was evaluated for the most suitable candidates previously presented: PVC random copolymers containing, respectively, $40 \%$ of BA and EHA (PVC-40\% BA random copolymer and PVC$40 \%$ EHA random copolymer). As shown in Figure 3 , in both cases the material printing is not possible for temperatures below $160^{\circ} \mathrm{C}$. However, the increase of temperature results in a viscosity reduction, which should enable printing. Even so, it worth noting that the higher printing temperature is limited by the polymer thermal stability, as is discussed in the last part of the paper. Therefore, the printing temperature is a key factor to optimize, reaching a compromise between the suitable viscosity and the thermal stability.

\subsection{Effect of acrylate concentration}

Facing the difficulties of tailoring the viscosity by increasing temperature, the variation of the acrylate

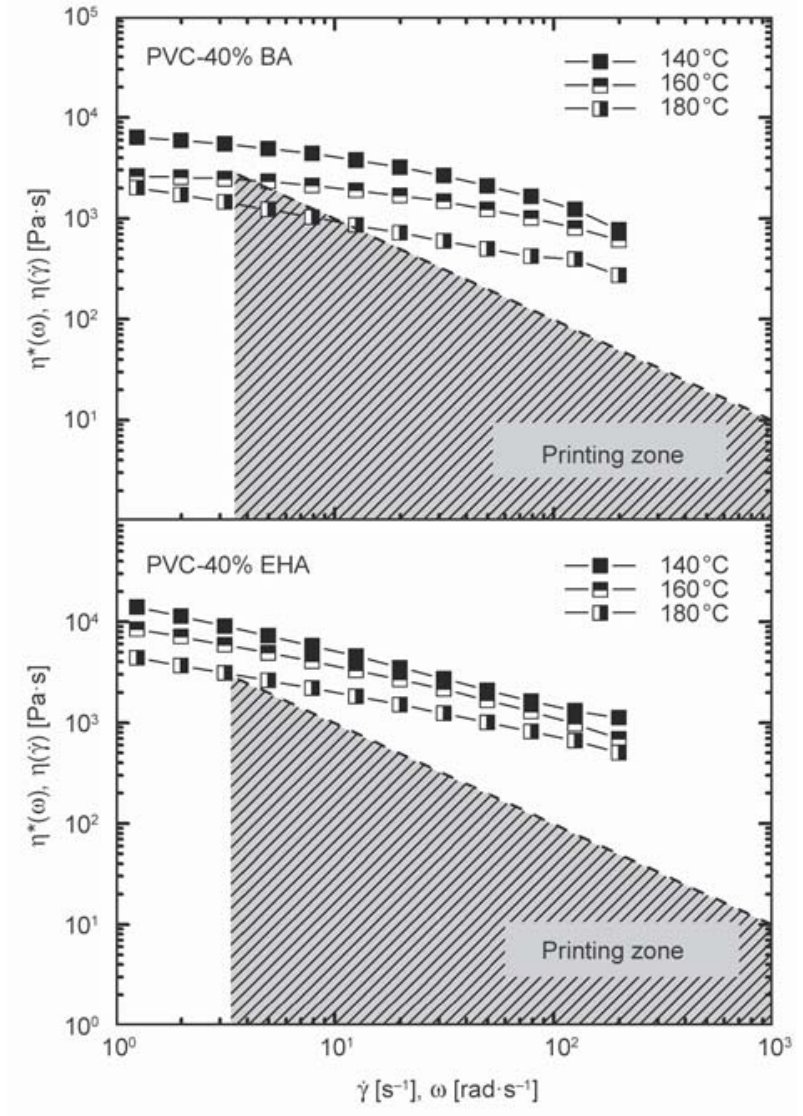

Figure 3. Complex viscosity, $\eta^{*}(\omega)$ as a function of frequency at the indicated temperatures for PVC- $40 \%$ BA and PVC-40\% EHA random copolymers. The line marks the postulated continuous flow viscosity, $\eta(\dot{\gamma})$ as a function of the shear rate for the maximum pressure of $8.8 \cdot 10^{5} \mathrm{~Pa}$ in the $3 \mathrm{D}$ printing machine (see text). For viscosities above the grey area, printing is not possible.

amount in the PVC-acrylate copolymers was studied as a synthetic alternative to produce such effect. Thus, other copolymers were synthesized, using the procedure mentioned in the Experimental Part, but varying the acrylate content from 20 to $50 \%$. The viscosity results of both PVC-BA and PVC-EHA random copolymers are shown in Figure 4. As expected, for low acrylate contents (20 and 30\%) the plasticizing effect is not high enough to permit the material printing. In contrast, high amounts of acrylates produce sufficient viscosity reduction to be inside the theoretically estimated printing limits (Figure 4). However, it is to be noted that the plasticizing effect of butyl acrylate is more noticeable than that of EHA monomer which hardly approaches printing region for the sample PVC-50\% EHA. In our opinion, this effect can be attributed to the not completely random feature of these copolymers, as suggested by the DMTA results (Figure 1d), which can reduce the 


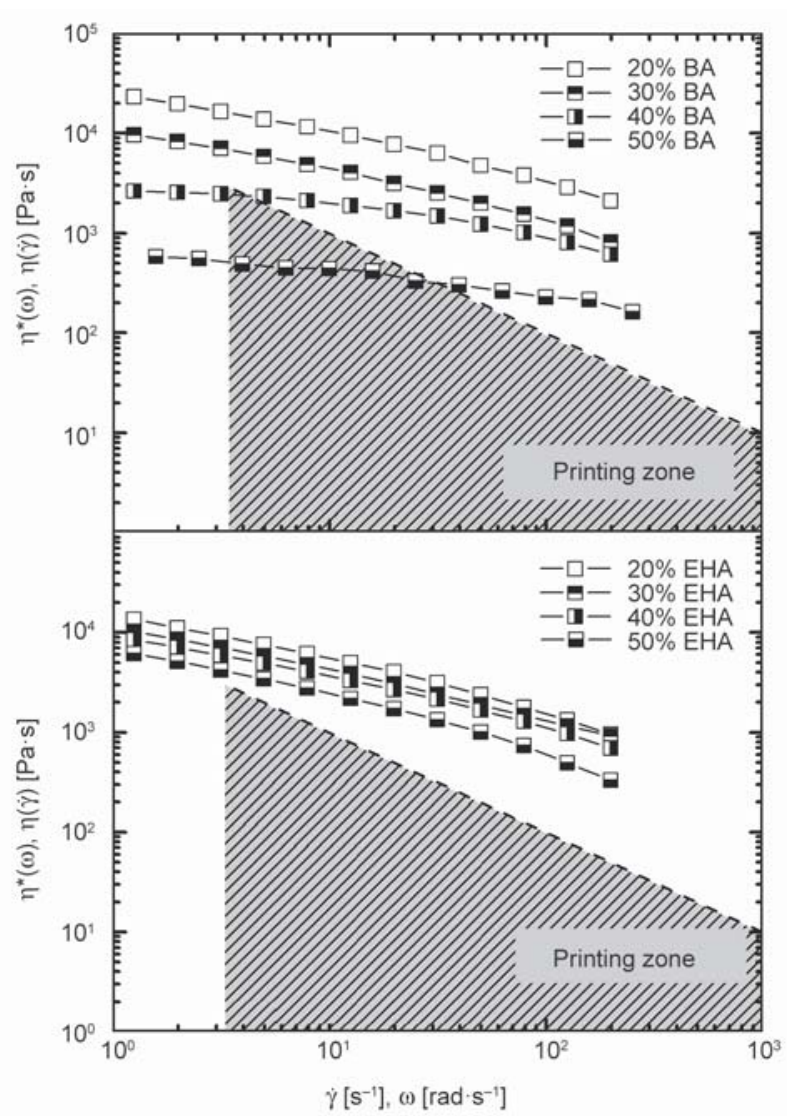

Figure 4. Effect of the acrylate concentration on the viscosity of PVC-BA and PVC-EHA copolymers materials and corresponding comparison with the limit of the viscosity for printing, marked by the grey zone.

\begin{tabular}{|l|c|l|c|}
\hline \multicolumn{4}{|c|}{ Feasibility of samples for printing } \\
\hline PVC-30\% BA & No & PVC-30\% EHA & No \\
\hline PVC-40\% BA & Yes & PVC-40\% EHA & No \\
\hline PVC-50\% BA & Yes & PVC-50\% EHA & - \\
\hline
\end{tabular}

a)

b)
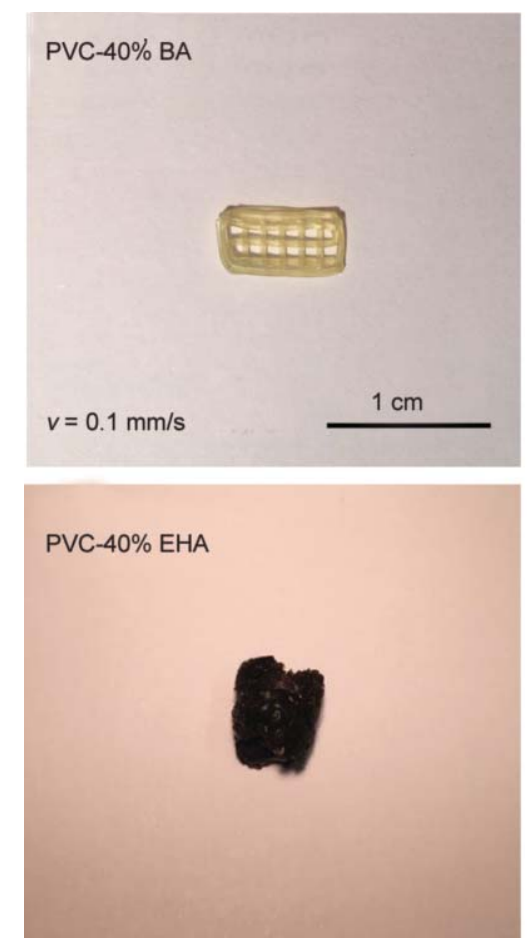

plasticizing effect in comparison with the BA counterpart.

The estimated printing capacities, derived from the rheological results of Figure 4, were compared with $3 \mathrm{D}$ printing experiments. The table presented in Figure 5 summarizes the printing performance at $160^{\circ} \mathrm{C}$. As predicted, samples containing acrylate amounts up to $30 \%$ were unable to be printed do to the high viscosity of materials. For samples containing higher amounts, two different behaviours were observed. As shown in Figure 5, PVC-BA random copolymers were successfully printed, being the PVC-50\% BA capable to reach printing velocities up to $2.2 \mathrm{~mm} / \mathrm{s}$. In contrast, PVC-EHA copolymers showed problems when printing. With the maximum pressure, it was not actually possible to extrude PVC-40\% EHA through the needle and it resulted in the material degradation inside the barrel, as can be seen in Figure $5 \mathrm{~b}$.

On the other hand, PVC-50\% EHA random copolymer brought about a material filament, instead of the programmed scaffold shape. The observed effects were predictable according to the rheological results of Figure 5. PVC-40\% EHA random copolymer displays excessively high viscosities. In the case of PVC-50\% EHA copolymer, viscosities at the lowest shear rates lie close to the limit for extrusion in our $3 \mathrm{D}$ printing device. The high viscosities result in a very low extrusion velocity which mismatches minimum 
printing velocity of the $3 \mathrm{D}$ printer. Consequently, once the material is stacked to the printing plate, the material filament is stretched due to the motion of the $3 \mathrm{D}$ printing needle, impeding the formation of the programed shape. In addition, the printing velocities achieved in the 3D Printing machine (Figure 5) matched with the viscosity values given in Figure 4: The lower the viscosity is, the higher is the printing velocity, as is the case of $\mathrm{PVC}-50 \% \mathrm{EHA}$ random copolymer.

\subsection{Cooling and material consolidation}

In conjunction with adequate viscosity, polymers should show a sound layer adhesion leading to good weld strength of the manufactured object or piece [7, $10,11]$. As pointed out by Miegler and coworkers [24], this is related to the capacity of the polymer chains for interlayer diffusion, which is only possible in the terminal or flow viscoelastic region. Simulation studies on chain diffusion at polymer interfaces, carried out by Ge et al. [25] showed that good welding is only accomplished for welding times $3 \cdot 10^{6}$ $\lambda_{0} \mathrm{~s}$, where $\lambda_{0}$ is a time which accounts for the motion of the monomers. Since the glassy state is associated to the local motions of monomers, lastingness of the order of million times higher should correspond to the terminal or flow viscoelastic zone. This zone is characterized by a predominance of the viscous modulus with respect to the elastic modulus, $G^{\prime \prime}>G^{\prime}$, which implies a dissipation of energy associated to the motion of the polymer chain as a whole, instead of the local motions of the glassy state or the segmental motions of the rubbery state. Only when the chains are able to diffuse at a large scale are entanglements between layers possible. Therefore, polymer melts apt for additive manufacturing should show a terminal viscoelastic zone at the temperatures involved in the manufacturing process.

Moreover, the temperature of the successive layers decreases with time and, therefore, dynamic viscoelastic measurements at different temperatures were carried out with the most promising samples, in order to determine the terminal viscoelastic zone, and its relaxation time $(\tau)$. Figure 6 shows the elastic and loss moduli results as a function of frequency for PVC-40\% BA random copolymer, as representative of the other random copolymers. The results shown in Figure 6 indicate that the terminal zone (at which $G^{\prime \prime}>G^{\prime}$ ) shifts to higher frequencies as the temperature is increased. The limit of the terminal or flow

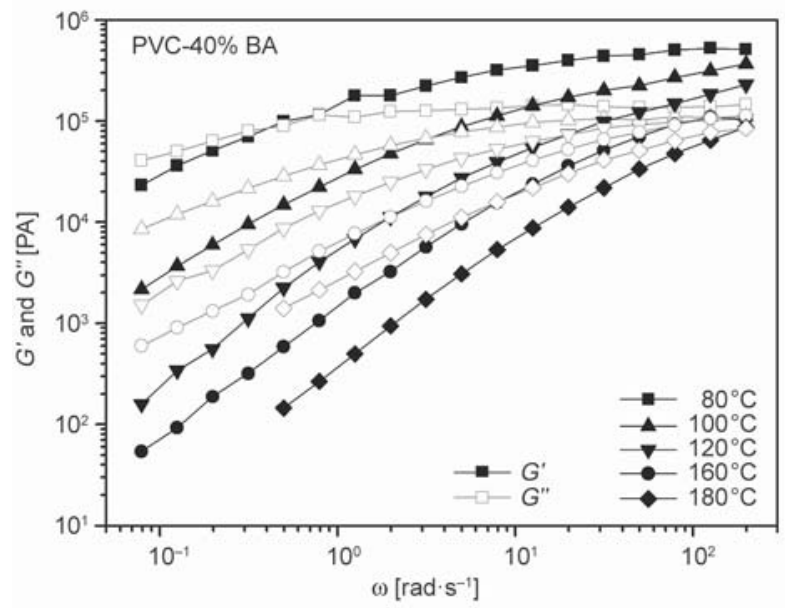

Figure 6. Elastic modulus, $G^{\prime}$ and viscous modulus, $G^{\prime \prime}$, as a function of frequency at different temperatures for PVC- $40 \%$ BA random copolymer. The relaxation time $\tau$ is defined as the inverse of the frequency at which $G^{\prime}=G^{\prime \prime}$ (see text).

zone is marked by the crossing frequency, $\omega x$, at which $G^{\prime}=G^{\prime \prime}$. The inverse of $\omega x$ is the relaxation time, $\tau=1 / \omega x$, that has the following physical meaning, in the context we are dealing with: For times $t>\tau$ the mobility of the chains corresponds to that expected for the terminal viscoelastic zone, so allowing chain diffusion and adhesion between layers, whereas for times $t<\tau$ the necessary time for adhesion is not accomplished.

The evaluation of $\tau$ from the results of Figure 6 indicates that the time for adhesion varies from $3.8 \cdot 10^{-3} \mathrm{~s}$, at $T=160^{\circ} \mathrm{C}$, to $2.5 \mathrm{~s}$ at $T=100^{\circ} \mathrm{C}$, so for an eventual contact time of $1 \mathrm{~s}$ adhesion would take place at $160^{\circ} \mathrm{C}$, but not at $100^{\circ} \mathrm{C}$. This outcome is relevant, because there is a temperature decrease of the polymer melt as welding between layers takes place. This temperature decrease has been evaluated by Seppälä and Migler [26] for an ABS copolymer, typically used in additive manufacturing. Unfortunately, evaluating the temperature profiles of our pioneering PVC-acrylate random copolymers is currently out of the scope of our work.

Notwithstanding, our dynamic viscoelastic measurements can provide us valuable information about the welding process. Thus, we have demonstrated that the characteristic time varies with the acrylate type and content. As shown in Figure 7a, the storage and loss moduli crossovers for PVC-EHA copolymers occur at lower frequencies than the corresponding crossovers of PVC-BA counterparts, for tests carried out at $T=100^{\circ} \mathrm{C}$. In both, PVC-EHA and PVC-BA copolymers, the increase in the acrylate content results in 

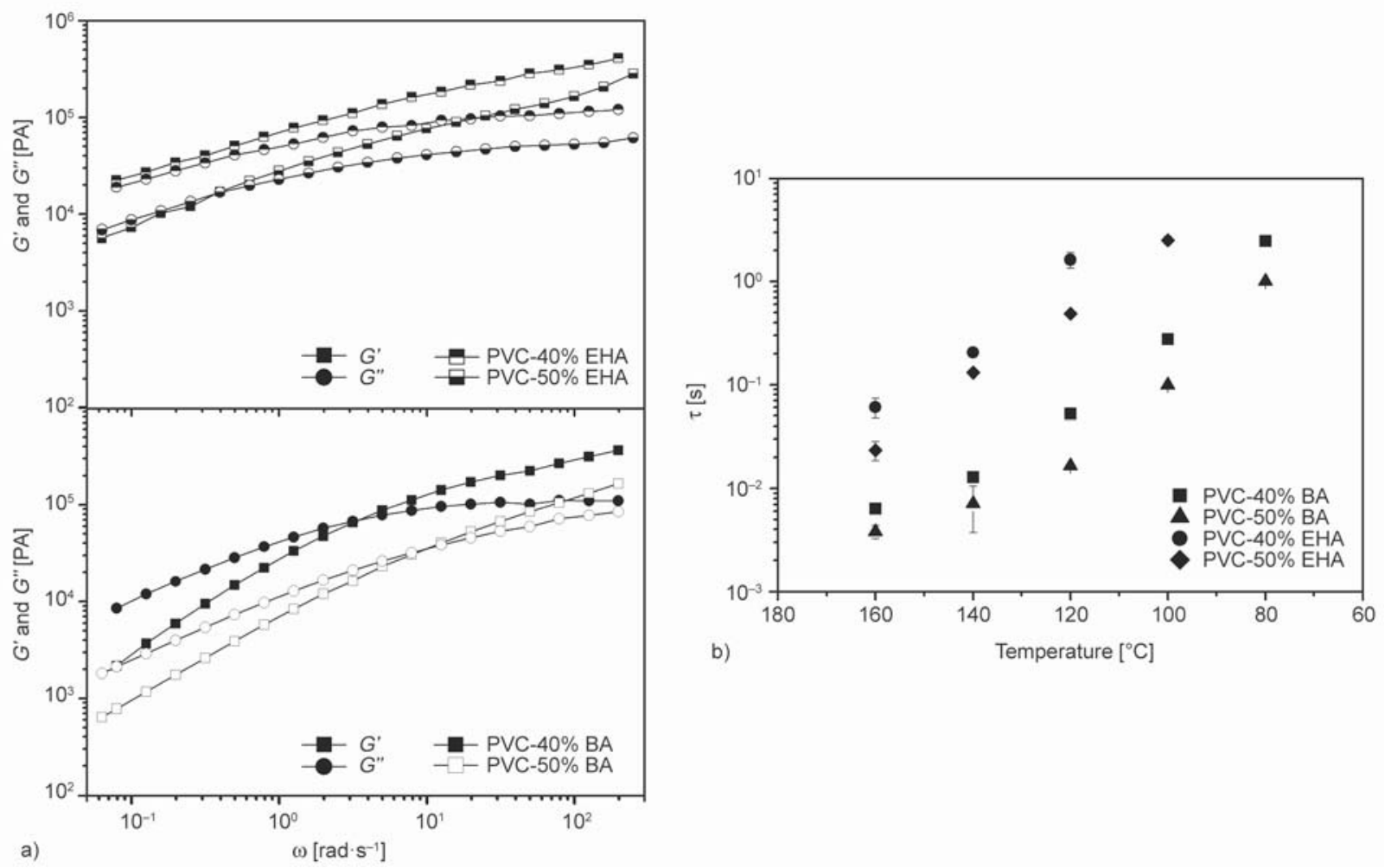

Figure 7. a) Elastic modulus, $G^{\prime}$, and viscous modulus, $G^{\prime}$, as a function of frequency for PVC-BA and PVC-EHA random copolymers at $100^{\circ} \mathrm{C}$ and $\mathrm{b}$ ) variation of the relaxation time $(\tau)$ with temperature. $\tau$ is defined as explained in Figure 6.

the shifting of the crossover point to higher frequencies. The combined effect of acrylate content and temperature can be clearly seen in Figure $7 b$, where the variation of the relaxation time $(\tau)$ with temperature for different samples is depicted. The plot shows that the incorporation of butyl acrylate monomer in the formulation produces lower characteristic times than the incorporation of 2-ethylhexilacrylate in the temperature range studied. In both cases, the incorporation of higher acrylate amounts to the respective copolymers results in a reduction of the relaxation time $\tau$. We recall that reducing $\tau$ results in easiness of the adhesion process, because chains diffusion between layers is allowed as long as the contact time is bigger than $\tau$.

The adhesion process was qualitatively evaluated by optical microscopy. The results are shown in Figure 8. The micrograph of the PVC-40\% BA sample (Figure 8a) shows no welding for the printed object, resulting in the failure of the specimen. In contrast, PVC-50\% BA (Figure 8b) shows a well welded specimen, as corresponds to a sample which has better conditions for interlayer chain diffusion, owed to the low value of its relaxation time $\tau$ (see Figure 7). This good adhesion was confirmed cutting transversally the welding zone and analysing it by optical microscopy.
The micrograph (Figure 8c) clearly shows that the interpenetration and welding between consecutive layers was achieved.

\subsection{Thermal stability of PVC-acrylate random copolymers}

Although the $3 \mathrm{D}$ printing process was achievable with the samples which fulfilled the adequate rheological features, one additional problem which was observed to arise in the investigated PVC-acrylate copolymers at 'a priori' suitable temperatures, was thermal degradation. The incorporation of acrylates to the PVC structure results in a reduction of the thermal stability of materials. This is an issue that should be contemplated, since the reduction of the thermal stability limits the maximum temperature at which the material can be printed, as well as the residence time of materials at high temperatures inside the barrel of the cartridge. Although the geometry of the 3D printer (capacity of $10 \mathrm{~cm}^{3}$ ) permits higher amounts of materials, in the framework of our study, the maximum material load was $3 \mathrm{~g}$, which implies a residence time of 2.5 hours. However, the material degradation occurs much faster, limiting the operation time and the dimensions of the specimens that can be printed. Thus, isothermal TGA measurements were 


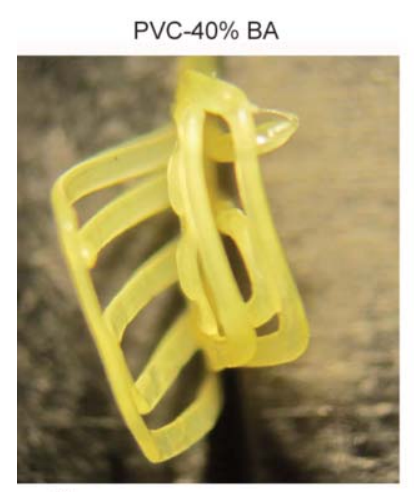

a)

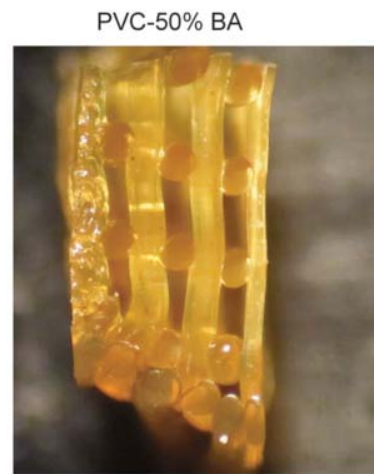

b)

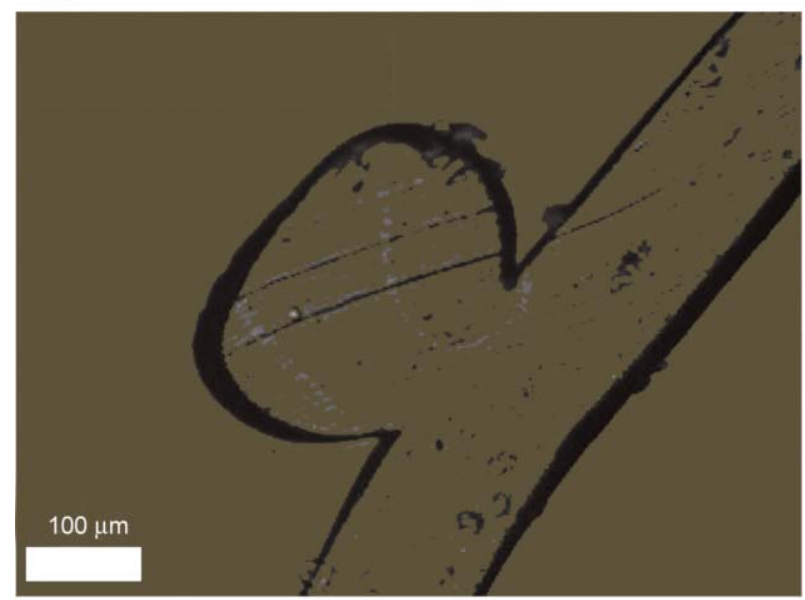

c)

Figure 8. Microscopy analysis of interlayer adhesion. a) PVC- $40 \%$ BA copolymer b) PVC- $50 \%$ BA copolymer and c) microphotography of a cross section of printed PVC-50\% BA copolymer.

carried out, in order to determine the maximum residence times without degradation at different temperatures. The results are presented in Figure 9. As can be observed in the isothermal thermogram at $160^{\circ} \mathrm{C}$, the copolymers show little mass reduction during the first 45 minutes. From that point on, the PVC-50\% BA random copolymer, the most promising sample in terms of 3D printability, starts to degrade. The other copolymers show a higher stability and remain almost unaltered to times nearly $70 \mathrm{~min}$. It is to be noted that the thermal stability decreases as the acrylate content increases, being the PVC-BA copolymers less stable than their PVC-EHA counterparts. In addition to this, the increase of temperature brings about a huge effect in the thermal stability of the copolymers, as could be expected. For instance, the degradation onset of PVC-50\% BA random copolymer varies from $45 \mathrm{~min}$ at $160^{\circ} \mathrm{C}$ to just $10 \mathrm{~min}$ at $180^{\circ} \mathrm{C}$. Consequently, although increasing temperature benefits 3D printing in terms of viscosity reduction, printing temperatures above $160^{\circ} \mathrm{C}$ are

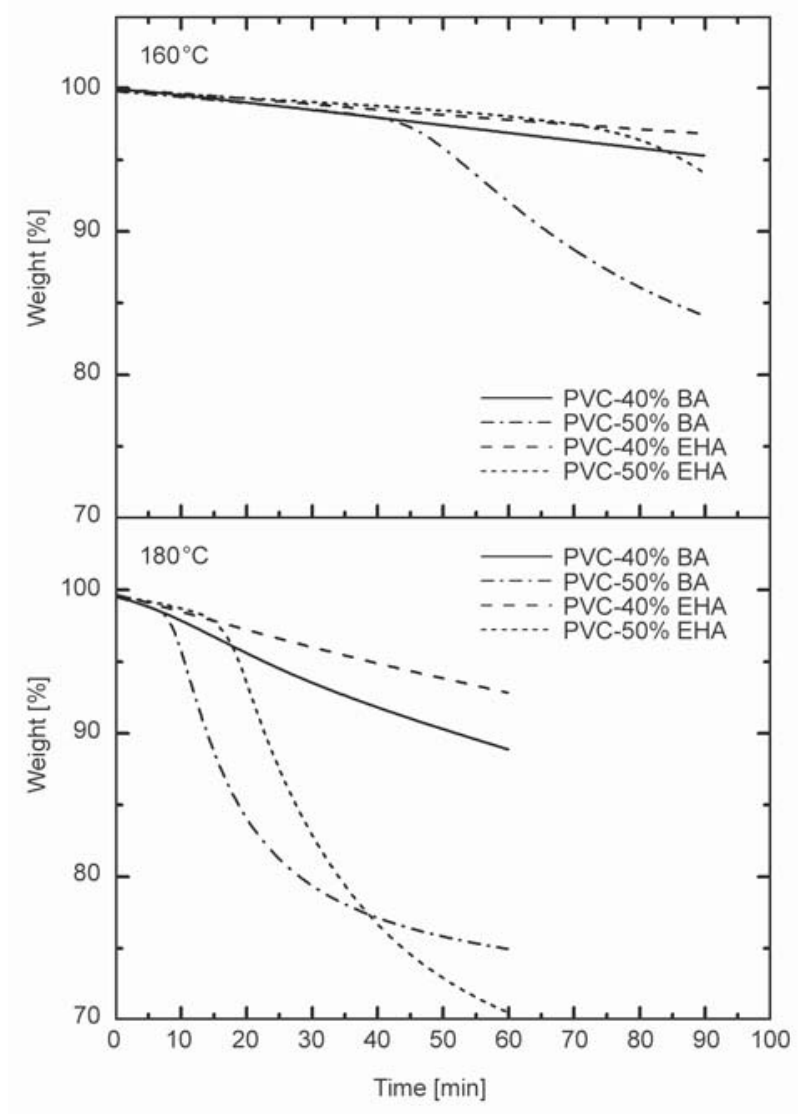

Figure 9. Isothermal TGA measurements for thermal stability analysis of the indicated copolymers at 160 and $180^{\circ} \mathrm{C}$.

unworkable due to the thermal degradation of the copolymers.

Certainly, this also affects the 3D printed specimen shape and appearance. As can be seen in Figure 10, PVC-50\% BA random copolymer allows 3D printing of several layers with a good space resolution and low yellowing. However, as the residence time increases $(30 \mathrm{~min})$, the $3 \mathrm{D}$ printed specimen acquires a brown color, indicating severe degradation. At long residence times (>50 $\mathrm{min}$ ), the material is so degraded that is unable to reproduce the programmed shape and it presents a blown-to-black color.

\section{Conclusions}

Continuous flow viscosity data and dynamic viscoelastic results analysed at the light of a basic hydrodynamic approach and considering the mobility of polymer chains for interlayer adhesion, allow establishing some prerequisites for a feasible extrusion-based 3D printing of PVC-acrylate copolymers. In the study, which is also valid for other amorphous polymers or for polymers having a low degree of 


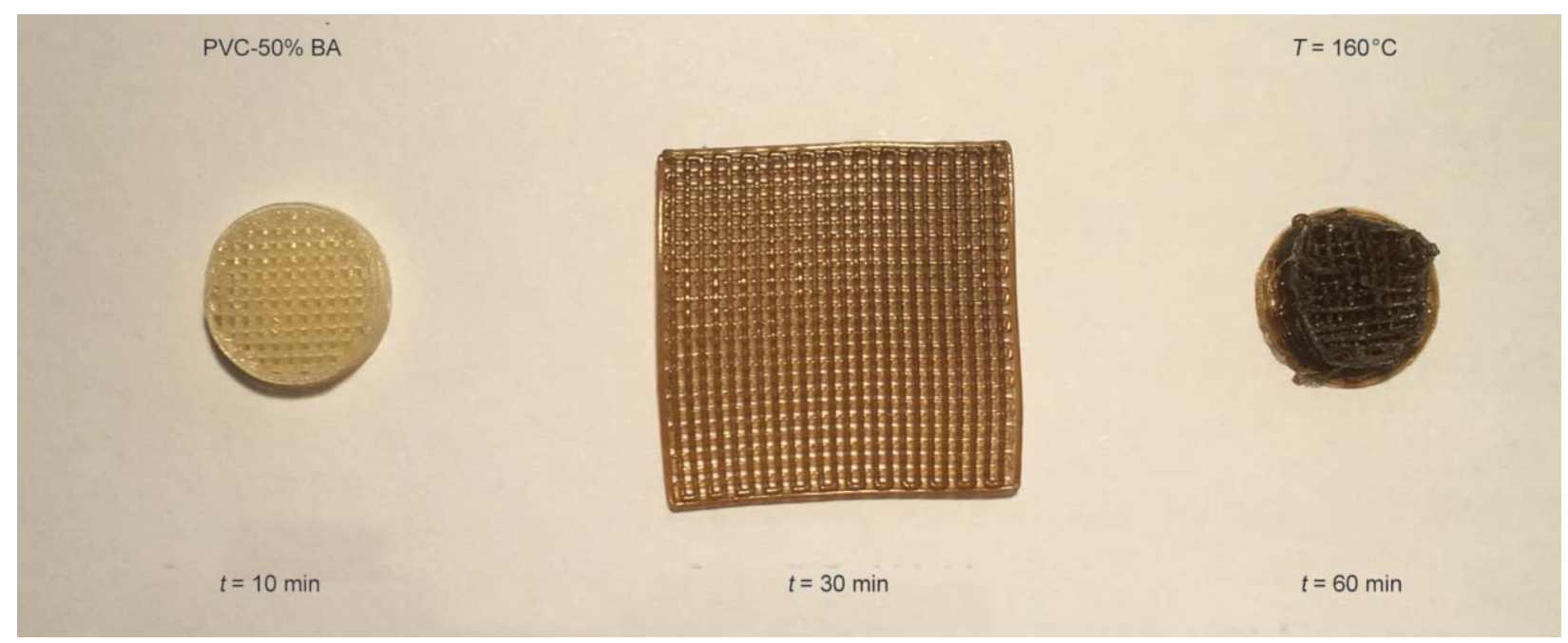

Figure 10. Time evolution of the 3D printed PVC-50\% BA copolymer coloration at $160^{\circ} \mathrm{C}$.

crystallinity, it is demonstrated that PVC-BA random copolymers obtained by Single Electron TransferDegenerative Chain Transfer Living Radical Polymerization (SET-DTLRP) possesses the most adequate rheological features to be 3D printed. Among the different formulations studied, PVC-50\% BA random copolymer is particularly satisfactory to elaborate objects by additive manufacturing, as it satisfies both extrusion and welding conditions. However, the study of the thermal degradation of this copolymer shows that there are limits, which affect the temperature and residence time of the elaboration process. Our analysis is focused on the results obtained with PVC-acrylate copolymers and a 3D printing device that possesses certain established characteristics (maximum pressure, needle diameter etc.), but shows a way to avoid trial and error when using other polymers and $3 \mathrm{D}$ printing machines

\section{Acknowledgements}

This work was financial supported by ERCROS S.A. and by UPV/EHU (UFI 11/56) and GIC IT-586-13 (Basque Government). Authors are also grateful by financial support of Programa Red guipuzcoana de Ciencia, Tecnología e Innovación 2017 (DFG). R.H.A. also acknowledges UPV/EHU for the grant 'ayudas para la especialización de personal investigador del vicerrectorado de investigación'.

\section{References}

[1] Ligon S. C., Liska R., Stampfl J., Gurr M., Mülhaupt R.: Polymers for 3D printing and customized additive manufacturing. Chemical Reviews, 117, 10212-10290 (2017).

https://oi.org/10.1021/acs.chemrev.7b00074
[2] Bikas H., Stavropoulos P., Chryssolouris G.: Additive manufacturing methods and modelling approaches: A critical review. International Journal of Advanced Manufacturing Technology, 83, 389-405 (2016). https://doi.org/10.1007/s00170-015-7576-2

[3] Gao W., Zhang Y., Ramanujan D., Ramani K., Chen Y., Williams C. B., Wang C. C. L., Shin Y. C., Zhang S., Zavattieri P. D.: The status, challenges, and future of additive manufacturing in engineering. Computer-Aided Design, 69, 65-89 (2015). https://doi.org/10.1016/j.cad.2015.04.001

[4] Turner B. N., Strong R., Gold S. A.: A review of melt extrusion additive manufacturing processes: I. Process design and modeling. Rapid Prototyping Journal, 20, 192-204 (2014).

https://doi.org/10.1108/RPJ-01-2013-0012

[5] Ozbolat I. T., Hospodiuk M.: Current advances and future perspectives in extrusion-based bioprinting. Biomaterials, 76, 321-343 (2016). https://doi.org/10.1016/j.biomaterials.2015.10.076

[6] Ciacci L., Passarini F., Vassura I.: The European PVC cycle: In-use stock and flows. Resources, Conservation and Recycling, 123, 108-116 (2017).

https://doi.org/10.1016/j.resconrec.2016.08.008

[7] McIlroy C., Olmsted P. D.: Disentanglement effects on welding behaviour of polymer melts during the fusedfilament-fabrication method for additive manufacturing. Polymer, 123, 376-391 (2017).

https://doi.org/10.1016/j.polymer.2017.06.051

[8] Lee J. M., Yeong W. Y.: A preliminary model of timepressure dispensing system for bioprinting based on printing and material parameters. Virtual and Physical Prototyping, 10, 3-8 (2015).

https://doi.org/10.1080/17452759.2014.979557

[9] Sarker M., Chen X. B.: Modeling the flow behavior and flow rate of medium viscosity alginate for scaffold fabrication with a three-dimensional bioplotter. Journal of Manufacturing Science and Engineering, 139, 81002/181002/14 (2017).

https://doi.org/10.1115/1.4036226 
[10] Davis C. S., Hillgartner K. E., Han S. H., Seppala J. E.: Mechanical strength of welding zones produced by polymer extrusion additive manufacturing. Additive Manufacturing, 16, 162-166 (2017).

https://doi.org/10.1016/j.addma.2017.06.006

[11] Levenhagen N. P., Dadmun M. D.: Bimodal molecular weight samples improve the isotropy of 3D printed polymeric samples. Polymer, 122, 232-241 (2017).

https://doi.org/10.1016/j.polymer.2017.06.057

[12] Levenhagen N. P., Dadmun M. D.: Interlayer diffusion of surface segregating additives to improve the isotropy of fused deposition modeling products. Polymer, in press (2018).

https://doi.org/10.1016/j.polymer.2018.01.031

[13] Wypych G.: Principles of thermal degradation. in 'PVC degradation and stabilization' (ed.: Wypych G.) ChemTech Publishing, Toronto, 79-165 (2015).

https://doi.org/10.1016/B978-1-895198-85-0.50006-6

[14] Calafel I., Muñoz M. E., Santamaría A., Boix M., Conde J. I., Pascual B.: The effect of crystallites on the rheological properties of microphase-separated PVC-PBAPVC triblock copolymers obtained by single electron transfer-degenerative chain transfer living radical polymerization. Journal of Vinyl and Additive Technology, 21, 24-32 (2015).

https://doi.org/10.1002/vnl.21397

[15] Calafel I., Muñoz M. E., Santamaría A., Boix M., Conde J. I., Pascual B.: PVC/PBA random copolymers prepared by living radical polymerization (SET-DTLRP): Entanglements and chain dimensions. European Polymer Journal, 73, 202-211 (2015).

https://doi.org/10.1016/j.eurpolymj.2015.10.013

[16] Rosen B. M., Percec V.: Single-electron transfer and single-electron transfer degenerative chain transfer living radical polymerization. Chemical Reviews, 109, 5069-5119 (2009).

https://doi.org/10.1021/cr900024j

[17] Percec V., Popov A. V., Ramirez-Castillo E., Weichold O.: Living radical polymerization of vinyl chloride initiated with iodoform and catalyzed by nascent $\mathrm{Cu}^{0} /$ tris (2-aminoethyl)amine or polyethyleneimine in water at $25^{\circ} \mathrm{C}$ proceeds by a new competing pathways mechanism. Journal of Polymer Science Part A: Polymer Chemistry, 41, 3283-3299 (2003).

https://doi.org/10.1002/pola.10937

[18] Percec V., Popov A. V., Ramirez-Castillo E., Coelho J. F. J., Hinojosa-Falcon L. A.: Phase transfer catalyzed single electron transfer-degenerative chain transfer mediated living radical polymerization (PTC-SET-DTLRP) of vinyl chloride catalyzed by sodium dithionite and initiated with iodoform in water at $43^{\circ} \mathrm{C}$. Journal of Polymer Science Part A: Polymer Chemistry, 43, 779-788 (2005).

https://doi.org/10.1002/pola.20544
[19] Coelho J. F. J., Simões P. N., Mendonça P. V., Fonseca A. C., Gil M. H.: Thermal characterization of poly(vinyl chloride) samples prepared by living radical polymerization: Comparison with poly(vinyl chloride) prepared by free radical polymerization. Journal of Applied Polymer Science, 109, 2729-2736 (2008).

https://doi.org/10.1002/app.28318

[20] Coelho J. F. J., Carreira M., Popov A. V., Gonçalves P. M. O. F., Gil M. H.: Thermal and mechanical characterization of poly(vinyl chloride)- $b$-poly(butyl acrylate)- $b$-poly(vinyl chloride) obtained by single electron transfer - Degenerative chain transfer living radical polymerization in water. European Polymer Journal, 42, 2313-2319 (2006).

https://doi.org/10.1016/j.eurpolymj.2006.05.023

[21] Coelho J. F. J., Carreira M., Gonçalves P. M. O. F., Popov A. V., Gil M. H.: Processability and characterization of poly(vinyl chloride)- $b$-poly( $n$-butyl acrylate)- $b$-poly (vinyl chloride) prepared by living radical polymerization of vinyl chloride. Comparison with a flexible commercial resin formulation prepared with PVC and dioctyl phthalate. Journal of Vinyl and Additive Technology, 12, 156-165 (2006).

https://doi.org/10.1002/vnl.20088

[22] Bird R. B., Curtiss C. F., Armstrong R. C., Hassager O.: Dynamics of polymeric liquids. Wiley, New York (1977).

[23] Venkatraman S., Okano M.: A comparison of torsional and capillary rheometry for polymer melts: The CoxMerz rule revisited. Polymer Engineering and Science, 30, 308-313 (1990). https://doi.org/10.1002/pen.760300508

[24] Seppala J. E., Han S. H., Hillgartner K. E., Davis C. S., Migler K. B.: Weld formation during material extrusion additive manufacturing. Soft Matter, 13, 6761-6769 (2017). https://doi.org/10.1039/C7SM00950J

[25] Ge T., Grest G. S., Robbins M. O.: Tensile fracture of welded polymer interfaces: Miscibility, entanglements, and crazing. Macromolecules, 47, 6982-6989 (2014). https://doi.org/10.1021/ma501473q

[26] Seppälä J. E., Migler K. B.: Infrared thermography of welding zones produced by polymer extrusion additive manufacturing. Additive Manufacturing, 12, 71-76 (2016).

https://doi.org/10.1016/j.addma.2016.06.007 